\title{
The Moss Flora of Kocaçay Delta (Karacabey-Bursa) Floodplain Forest in Turkey
}

\author{
Serhat URSAVAŞ*1 iD, Tamer KEÇELİ ${ }^{2}$ iD \\ ${ }^{I}$ Department of Forest Engineering, Faculty of Forestry, Çankırl Karatekin University, 18200, Çankırl, \\ TURKEY \\ ${ }^{2}$ Department of Biology, Faculty of Science, Çankırı Karatekin University, Çankırı, 18200, TURKEY
}

\begin{tabular}{lll}
\hline Received: 24.01.2019 & Revised: 04.02.2019 & Accepted: 15.02.2019
\end{tabular}

\begin{abstract}
In this study, the moss flora of Kocaçay Delta (Bursa-Karacabey) floodplain forest in Turkey were investigated in 2017. Three hundred eighty three moss samples, were collected and identified from Kocaçay-Delta. A total of 112 taxa belonging to 25 families and 60 genera were determined. In terms of taxa number, the riches six families are; Pottiaceae (26), Brachytheciaceae (15), Bryaceae (10), Hypnaceae (8), Orthotrichaceae (6), Polytrichaceae (5). In terms of genera number, the riches five genera are; Tortula (7), Hypnum (6), Orthotrichum (6), Weissia (5), Ptychostomum (5). 13 moss taxa are new record for (A1) square.
\end{abstract}

Key words: Moss, flora, Bursa, Karacabey, A1, Turkey.

\section{Kocaçay Deltası Longoz Ormanlarının (Karacabey-Bursa) Karayosunu Florası}

$\ddot{O} z$

Bu çalışmada, 2017 yılları arasında Kocaçay Deltası (Bursa-Karacabey) longoz ormanlarının karayosunu florası araştırılmıştır. Kocaçay Deltasından toplanan 383 karayosunu örneğinin incelenmesi sonucu; 25 familyaya ve 60 cinse ait 112 takson belirlenmiştir. Taksonlar açısından en zengin altı familya şu şekildedir; Pottiaceae (26), Brachytheciaceae (15), Bryaceae (10), Hypnaceae (8), Orthotrichaceae (6) ve Polytrichaceae (5)'dir. Takson sayısı bakımından en zengin beş cins; Tortula (7), Hypnum (6), Orthotrichum (6), Weissia (5) ve Ptychostomum (5). 13 karayosunu taksonu A1 karesi için yeni kayıttır.

Anahtar kelimeler: Karayosunu, flora, Bursa, Karacabey, A1, Türkiye.

* Corresponding author: serhatursavas@gmail.com

(C) 2019 All rights reserved / Tüm haklart saklıdır.

To cite this article: Ursavaş S. Keçeli T. 2019. The Moss Flora of Kocaçay Delta (Karacabey-Bursa) Floodplain Forests in Turkey. Anatolian Bryology. 5:1, 22-34.

(c) $)(1)$ ( ) This work is licensed under a Creative Commons Attribution-NonCommercial 4.0 International License. 


\section{Introduction}

Owing to rapid population growth and human activities, coastal areas have very intensively been used for tourism, agricultural purposes, the industry sector, and therefore, they have deteriorated irreparably. Coastal areas are also affected by some natural disasters and events, such as extreme storm waves, tsunamis, tides and sea level changes. Ecologically, sustainability of coastal areas is crucial. In particular, lagoon groups, which are constitute about 13 percent of the coasts in the world, are very valuable ecosystems (Saçın, 2010).

Wenger et al. (1990) accentuated the importance and primacy of the studies to characterize the useful structures and plant diversity of this forest owing to decrease in the area for the longoze forest ecosystem in Europe. Additionally, wooded wetlands as a forest stock are the important component to define the bryophyte biodiversity (Schuck et al., 1994; Kavgaci et al., 2007; Işın and Ursavaş, 2018). Even though Kocaçay Delta is a noteworthy area in terms of ecosystem variation, no bryo-floristic study have been conducted in this area until now.

\subsection{Study area}

Kocaçay Delta (Karacabey-Bursa-) Floodplain Forest has an area of 42.000 hectares. Kocaçay Delta, approximately 25 kilometers north of Bursa-Karacabey, is located on the southern coast of the Marmara Sea. Delta has a great importance for the natural life since there are two shallow lagoons named Dalyan and Arapçiftliği, floodplain forest, lake, swamp, large sand dunes in the area (URL1). There is a wildlife development area (140.000 ha) with hills covered with deciduous forests in the south of the delta (Eken et al., 2006). In this wildlife area, there are Celal Acar Wildlife Rescue and Rehabilitation Training Center (28.611 ha.), Karadere-Pheasant Production Station (annual capacity 5.000) and Orhaneli public hunting area (4.955 ha). Dalyan and Poyraz lakes, which are located in the western part of the Delta and have a total area of 194 hectares, are fed by Malic Creek. There are also reeds covering 600 ha area, floodplain forests consisting of ash, alder and willows spread over an area of 730 hectares, and a large dune band with a wide variety of flora in the western part of the Delta (URL2).

Kocaçay Delta ecosystem diversity in terms of importance in Turkey has a place (Figure 1). The floodplains have many different ecosystems, including open areas, meadows, pastures, dunes, especially deciduous and coniferous forest areas. The vegetation encountered in the area is as follows: Acer campestre, Alnus glutinosa, Arbutus unedo, Asplenium scolopendrium, Cardamine bulbifera, Carpinus orientalis, $C$. betulus, Castanea sativa, Centaurium erythraea, Cistus creticus, Cirsium hypoleucum, Cota tinctoria, Cornus sanguinea, Corylus avellana Erica arborea, Ficus carica, Fraxinus angustifolia, F. ornus, Daphne pontica, Lavandula pedunculata subsp. cariensis, Periploca graeca, Paliurus spina-christi, Sarcopoterium spinosum, Smilax excelsa, Ruscus aculeatus, Ruscus hypoglossum, Populus alba, Leucojum aestivum, Phillyrea latifolia, Hypericum calycinum, Hypericum perforatum, Quercus coccifera, , Q. frainetto, Q. pubescens, Q. cerris, Vitis sylvestris, Laurus nobilis, Pistacia terebinthus, Pteridium aquilinum, Pinus brutia, P. pinea, Styrax officinalis, Viburnum tinus, Olea eropaea, Calicotome villosa, Spartium junceum, Osyris alba, Hedera helix, Ilex colchica, Prunella vulgaris, Fagus orientalis, Populus tremula, Laurocerasus officinalis, Silene compacta, Tilia tomentosa, Mespilus germanica, Phytolacca americana, Sambucus nigra, Asplenium scolopendrium, Sorbus torminalis, Polypodium vulgare, Taxus baccata, Styrax officinalis. 


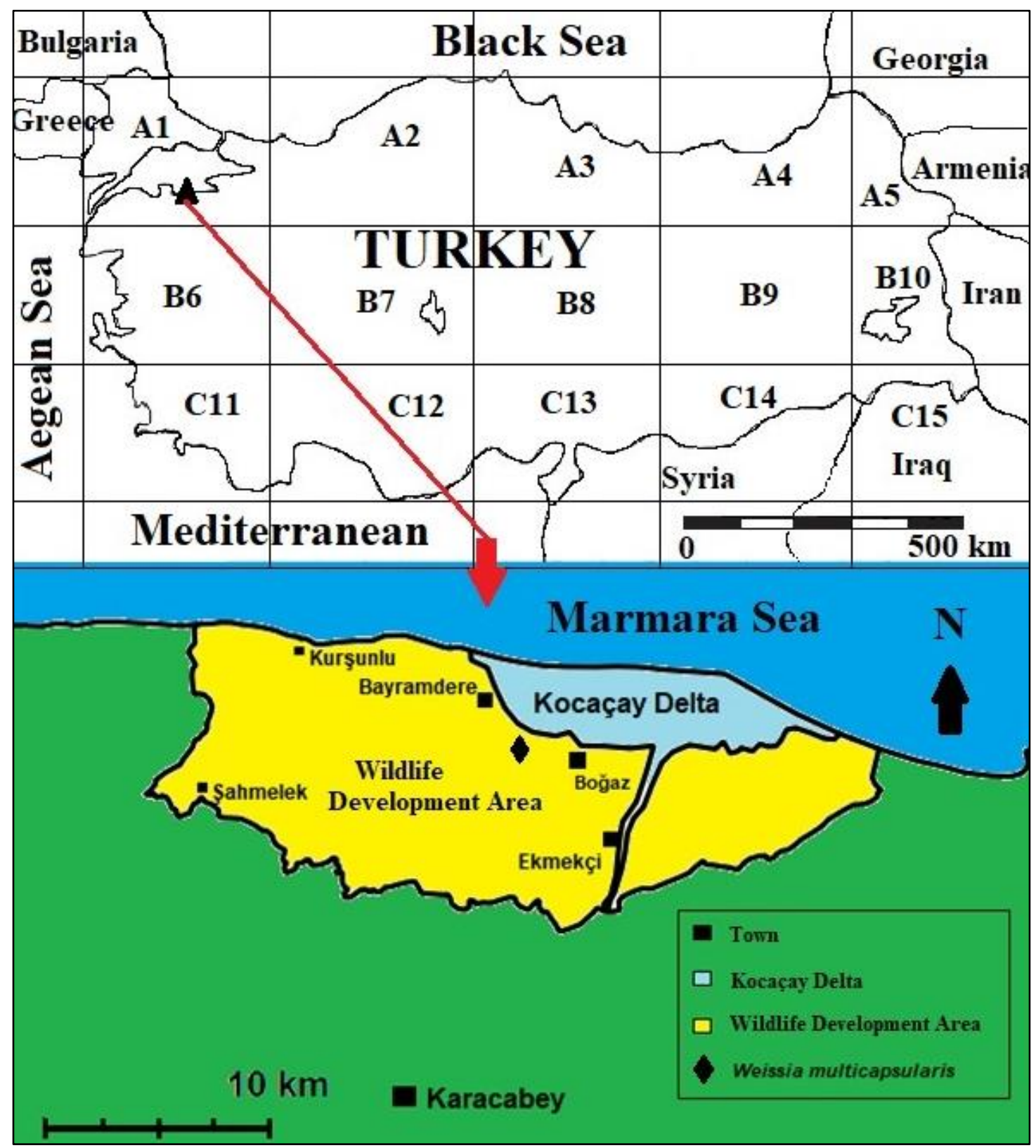

Figure 1. The location of the research area $(\mathbf{\Delta})$ according to the grid system of Turkey (Henderson, 1961), and locality of Weissia multicapsularis ( $\bullet$.

\section{Materials and Methods}

This study was carry out in Kocaçay delta longoze forest of Turkey in 2017. During the bryological survey held in the region of BursaKaracabey specimens were collected by $\mathrm{S}$. URSAVAŞ and T. KEÇELİ. A total of 383 specimens from 49 locality were collected and kept in the private Herbarium of Serhat Ursavas at Çankırı Karatekin University, Faculty of Forestry. Weisse multicapsularis with this study were identified as new records for Turkey (Ursavaş and Keçeli, 2018).
The moss samples were scrutinized with Leica EZ4 HD stereomicroscop and Olympus BX50 light microscope. Identifications were determined by consulting various keys (Lawton 1971; Crum, 1973; Crum and Anderson, 1981; Smith, 1980, 2004; Watson, 1981; Pedrotti, 2001, 2006; Heyn and Herrnstadt, 2004; Greven, 2003; Lüth, 2006a, 2006b, 2006c, 2007, 2008, 2009, 2010).

According to Henderson (1961) grid square, Kocaçay Delta floodplain forests are located in A1 square. Two bryophyte studies have been carried out in Bursa. The first study; was carried 
out by Çetin (1999) "The Moss Flora of the Uludağ National Park (Bursa/Turkey). The second study was carried out by Can Gözcü et al. (2018) "Epiphytic bryophyte vegetation of the Samanlı Mountains (Sakarya-Kocaeli-YalovaBursa) in Northwest Turkey".

\section{Findins}

\subsection{Abreviations:}

ш: Second record for Turkish bryophyte flora

*: New record for A1 grid square

**: New record for Bursa

U: Collection number for Serhat URSAVAŞ

Loc: Locality number

\subsection{List of collections site}

1) Longoz coast, 35T0624870, 4472397, Fraxinus angustifolia, sand-reeds field, $4 \mathrm{~m}$, 28.04.2017.

2) Longoz-Dalyan lake, 35T0626566, 4472511, Paliurus spina-christi, sand-reeds and bushes field, $3 \mathrm{~m}, 28.04 .2017$.

3) Longoz-bird observation tower, 35T0624120, 4472115, Fraxinus angustifolia, Alnus glutinosa, reeds and bushes field, $3 \mathrm{~m}$, 29.04.2017.

4) Longoze-northern part, 35T0622245, 4471895, Fraxinus angustifolia, swamp-reeds field, $5 \mathrm{~m}, 29.04 .2017$.

5) Longoze, 35T0621526, 4471631, Fraxinus angustifolia, Populus alba, openness area, 9 $\mathrm{m}$, 29.04.2017.

6) Dalyan lake, 35T0622123, 4471593, Fraxinus angustifolia, openness-reeds field, 3 $\mathrm{m}, 29.04 .2017$.

7) The inland swamp, 35T0620746, 4471574, Fraxinus angustifolia, Laurus nobilis, Paliurus spina-christi, reeds and bushes field, $5 \mathrm{~m}, 29.04 .2017$.

8) Hayırlar district, 35T0624636, 4463020, Pinus brutia, Quercus coccifera, Phillyrea latifolia, Paliurus spina-christi, Arbutus unedo, Erica arborea, 30 m, 30.04.2017.

9) Ballikaya district, 35T0625443, 4466986, Paliurus spina-christi, Styrax officinalis, Arbutus unedo, Quercus coccifera, Laurus nobilis, Cornus sanguinea, Pistacia terebinthus, $33 \mathrm{~m}, 30.04 .2017$.

10)Ekinli district, 35T0628370, 4469124, Tilia sp., Paliurus spina-christi, Styrax officinalis, Arbutus unedo, Quercus coccifera, Fraxinus ornus, Laurus nobilis, Cornus sanguinea, Carpinus orientalis, Pistacia terebinthus, Erica arborea, 47 m, 30.04.2017.

11)Arapçiftliği, 35T0629993, 4470062, Fraxinus angustifolia, swamp-reeds field, $0 \mathrm{~m}$, 01.05.2017.
12) Mesudiye district, 35T0634631, 4470177 , Phillyrea latifolia, Laurus nobilis, Ficus carica, seaside, $7 \mathrm{~m}, 01.05 .2017$.

13)Between Mesudiye and Ballikaya district, 35T0632003, 4470264, 18 m, Pinus pinea, Fraxinus ornus, Phillyrea latifolia, Styrax officinalis, Laurus nobilis, wooded area, 18 $\mathrm{m}, 01.05 .2017$.

14) South of longoze, 35T0625537, 4469238, Quercus cerris, Quercus pubescens, Phillyrea latifolia, Olea eropaea, Paliurus spina-christi, Styrax officinalis, Arbutus unedo, Arbutus andrachne, Quercus coccifera, Fraxinus ornus, Laurus nobilis, Cornus sanguinea, Carpinus orientalis, Pistacia terebinthus, Spartium junceum, Daphne pontica, Erica arborea, Forest and Pseudo-maquis, $12 \mathrm{~m}, 13.06 .2017$.

15)Boğazköy district, 35T0623334, 4469115, Quercus robur, Quercus cerris, Fraxinus angustifolia, Paliurus spina-christi, Forest area, $2 \mathrm{~m}, 13.06 .2017$.

16)Boğazköy district, 35T0623150, 4469223, Fraxinus angustifolia, Populus alba, Quercus cerris, Crataegus sp., Laurus nobilis, swamp area, $1 \mathrm{~m}, 14.06 .2017$.

17)Boğazköy district, 35T0623323, 4469347, Fraxinus angustifolia, Paliurus spina-christi, bush and grassland area, $0 \mathrm{~m}, 14.06 .2017$.

18)Boğazköy district, 35T0623415, 4469402, Fraxinus angustifolia, thickets, meadows, swamps, wetlands, $0 \mathrm{~m}, 14.06 .2017$.

19) South of Bogazköy, 35T0623117, 4468858, Castanea sativa, Carpinus betulus, Phillyrea latifolia, Styrax officinalis, Ilex colchica, Laurus nobilis, Daphne pontica, Ficus carica, Forest area, $25 \mathrm{~m}, 14.06 .2017$.

20) South of Bogazköy, 35T0623097, 4468713, Carpinus betulus, Castanea sativa, Styrax officinalis, Ilex colchica, Laurus nobilis, Daphne pontica, Forest area, $59 \mathrm{~m}$, 14.06.2017.

21)Celal Acar Wildlife Rescue and Rehabilitation Center, Ovakorusu Bear Shelter, 35T0620431, 4469093, Pinus pinea plantation forest, $12 \mathrm{~m}, 14.06,2017$.

22) Şahmelek district, 35T0605896, 4465671, Carpinus betulus, Fagus orientalis, $459 \mathrm{~m}$, 15.06.2017.

23)Between Şahmelek and Kurşunlu district, 35T0607293, 4467186, Fagus orientalis, Carpinus betulus, Alnus glutinosa, Castanea sativa, Populus tremula, Erica arborea, Laurocerasus officinalis, 504 m, 15.06.2017.

24)Between Şahmelek and Kurşunlu district, 35T0608350, 4466943, Fagus orientalis, Carpinus betulus, Castanea sativa, Alnus 
glutinosa, Populus tremula, Erica arborea, Daphne pontica, 498 m, 15.06.2017.

25)Between Şahmelek and Kurşunlu district, 35T0608379, 4466981, Fagus orientalis, Carpinus betulus, Castanea sativa, Alnus glutinosa, Populus tremula, Erica arborea, Daphne pontica, 487 m, 15.06.2017.

26)Between Şahmelek and Kurşunlu district, 35T0609283, 4466650, Castanea sativa, Fagus orientalis, Carpinus betulus, Daphne pontica, $448 \mathrm{~m}, 15.06 .2017$.

27)Between Şahmelek and Kurşunlu district, 35T0606795, 4468164, Fagus orientalis ormanı, Daphne pontica, 690 m, 15.06.2017.

28)Between Şahmelek and Kurşunlu district, 35T0606758, 4469698, Fagus orientalis ormani, Laurocerasus officinalis, Ilex colchica, Sambucus nigra, $769 \mathrm{~m}$, 15.06.2017.

29)Between Şahmelek and Kurşunlu district, 35T0607757, 4470829, Fagus orientalis, Carpinus betulus, Sambucus nigra, 567 m, 15.6.2017.

30)Kurşunlu district, 35T0607742, 4471178, Fagus orientalis, Carpinus betulus, Castanea sativa, Populus tremula, Erica arborea, Daphne pontica, Sambucus nigra, $436 \mathrm{~m}$, 15.06.2017.

31)Yeniköy district, 35T0611295, 4472668, Fagus orientalis, Castanea sativa, Carpinus betulus, $8 \mathrm{~m}, 15.06 .2017$.

32)Yarış district, 35T0615491, 4462380, Carpinus betulus, Sorbus torminalis, Daphne pontica, $389 \mathrm{~m}, 16.06 .2017$.

33)Güngörmez district, 35T0614686, 4464451, Fagus orientalis orman1, Quercus cerris, Quercus. frainetto, Carpinus betulus, Castanea sativa, Sorbus torminalis, Populus tremula, Erica arborea, Daphne pontica, 461 m, 16.06.2017.

34)Güngörmez district, 35T0615160, 4465961, Fagus orientalis ormani, Carpinus betulus, Castanea sativa, Sorbus torminalis, Populus tremula, Erica arborea, Daphne pontica, Sambucus ebulus, Hypericum calycinum, Cirsium hypoleucum, 452 m, 16.06.2017.

35)Between Güngörmez and Yarış district, 35T0614729, 4467594, Fagus orientalis ormanı, Carpinus orientalis, Castanea sativa, Sorbus torminalis, Laurocerasus officinalis, Erica arborea, Daphne pontica, 472 m, 16.06.2017.

36)Between Güngörmez and Yarış district, 35T0614860, 4468612, Fagus orientalis, Carpinus betulus, Castanea sativa, Sorbus torminalis, Laurocerasus officinalis, Erica arborea, Daphne pontica, 454 m, 16.06.2017.
37) Yarış district, 35T0614673, 4469097, Fagus orientalis orman1, Carpinus betulus, Castanea sativa, Sorbus torminalis, Laurocerasus officinalis, Erica arborea, Vaccinium arctostaphylos, $491 \mathrm{~m}$, 16.06.2017.

38)Çeşme district, 35T0616828, 4461887, Quercus cerris, Quercus frainetto, Carpinus betulus, Sorbus torminalis, Erica arborea, Daphne pontica, Cistus creticus, $387 \mathrm{~m}$, 16.06.2017.

39)Çamlıca district, 35T0626459, 4463108, Pinus brutia, Quercus pubescens, Q. cerris, Phillyrea latifolia, Pistacia terebinthus, Arbutus unedo, Laurus nobilis, Erica arborea, Daphne pontica, 46 m, 16.06.2017.

40)Wind Power Plant, 35T0633853, 4467980, Quercus pubescens, Q. cerris, Q. coccifera, Phillyrea latifolia, Styrax officinalis, Pistacia terebinthus, Pyrus elaeagniifolia, Paliurus spina-christi, Spartium junceum, 309 m, 16.06.2017.

41)Between Boğazköy and Yenice district, 35T0619771, 4469010, Pinus brutia, Quercus cerris, Q. frainetto, Q. coccifera, Acer campestre, Carpinus betulus, Styrax officinalis, Populus tremula, Paliurus spinachristi, Erica arborea, Cistus creticus, Phillyrea latifolia, Pistacia terebinthus, Arbutus unedo, Laurus nobilis, Erica arborea, Ruscus aculeatus, 44 m, 17.06.2017.

42)Bayramdere district, 35T0618415, 4467705 , Fagus orientalis, Carpinus betulus, Castanea sativa, Quercus frainetto, Q. cerris, Platanus orientalis, Alnus glutinosa, Acer campestre, Styrax officinalis, Populus tremula, Paliurus spina-christi, Erica arborea, Cistus creticus, Phillyrea latifolia, Laurus nobilis, Pistacia terebinthus, Arbutus unedo, Laurus nobilis, Erica arborea, $166 \mathrm{~m}, 17.06 .2017$.

43)Bayramdere dam pond, 35T0617857, 4466964, Fagus orientalis, Carpinus betulus, Castanea sativa, Quercus cerris, Platanus orientalis, Laurocerasus officinalis, Acer campestre, Styrax officinalis, Populus tremula, Paliurus spina-christi, Erica arborea, Laurus nobilis, Pistacia terebinthus, $244 \mathrm{~m}, 17.06 .217$.

44)Bayramdere dam pond, 35T0617244, 4465671, Quercus cerris, Q. frainetto, Fagus orientalis, Carpinus betulus, $325 \mathrm{~m}$, 17.06.2017.

45)Çeşme district, 35T0617182, 4466355, Fagus orientalis, Carpinus betulus, Castanea sativa, Erica arborea, Cistus creticus, $315 \mathrm{~m}$, 17.06.2017.

46) The northern part of Bayramdere, 35T0616657, 4470496, Castanea sativa, 
Carpinus betulus, Fagus orientalis, Platanus orientalis, Taxus baccata, Corylus avellana, Styrax officinalis, Laurocerasus officinalis, Laurus nobilis, Populus tremula, Arbutus unedo, Erica arborea, Sambucus ebulus, 152 $\mathrm{m}, 17.06 .2017$.

47)Bayramdere district, 35T0615043, 4470694, Fagus orientalis, Castanea sativa, Carpinus betulus, Platanus orientalis, Ficus carica, Pteridium aquilinum, Sorbus torminalis, Erica arborea, 344 m, 17.06.2017.

48)Bayramdere district, 35T0614407, 4471119, Fagus orientalis, Castanea sativa, Carpinus betulus, Daphne pontica, 430 m, 17.06.2017.

49)Between Yeniköy and Kurşunlu, 35T0613572, 4473027, Laurus nobilis, Styrax officinalis, 83 m, 17.06.2017.

\subsection{Plant list}

The bryoploristic list Nomenclature of the species follows Ros et al. (2013), Plášek et al. (2015) and Lara et al. (2016).

\section{BRYOPHYTA Schimp.}

Polytrichaceae Schwägr.

*Atrichum tenellum (Röhl.) Bruch \& Schimp.

Loc: 112, 120, on soil, U2854; U2853;

Atrichum undulatum (Hedw.) P. Beauv.

Loc: 107, 112, on soil, U2852; U2851;

Pogonatum aloides (Hedw.) P. Beauv.

Loc: 107, 112, 114, 118, on soil, U2857; U2856; U2858; U2855;

**Polytrichum formosum Hedw.

Loc: 112, on soil, U2849;

Polytrichum juniperinum Hedw.

Loc: 129, on rock, U2850;

Funariaceae Schwägr.

Funaria hygrometrica Hedw.

95, 100, on soil, U2835; U2836;

Grimmiaceae Arn.

Grimmia pulvinata (Hedw.) Sm.

Loc: 128, on soil, U2888;

Grimmia trichophylla Grev.

Loc: 122, on concrete, U2890; Loc: 103, 109, on rock, U2892; U2891;

**Grimmia lisae De Not.

Loc: 103, on rock, U2889;

Schistidium confertum (Funck) Bruch \& Schimp.

Loc: 122, on concrete, U2887;

Fissidentaceae Schimp.

**Fissidens bryoides Hedw.

Loc: 107, 109, 116, 117, 118, on soil, U2897; U2898; U2896; U2899; U2900;

**Fissidens taxifolius Hedw.

Loc: 103, 107, 125,126, 132, on soil, U2860; U2862; U2894; U2895; U2893;

*Fissidens viridulus var. viridulus (Sw. ex anon.) Wahlenb.
Loc: 92, 106, on soil, U2859; U2864;

**Fissidens viridulus var. incurvus (Starke ex Röhl.) Waldh.

Loc: 113, on soil, U2861;

Ditrichaceae Limpr.

*Ceratodon conicus (Hampe) Lindb.

Loc: 129, on rock, U2903;

Ceratodon purpureus (Hedw.) Brid.

Loc: 128, on rock, U2906;

**Ditrichum heteromallum (Hedw.) E.Britton

Loc: 94, 113, on soil, U2907; U2908;

**Pleuridium subulatum (Hedw.) Rabenh.

Loc: 108, on soil, U2911;

Dicranaceae Schimp.

**Dicranella heteromalla (Hedw.) Schimp.

Loc: on soil, U2902;

*Dicranella subulata (Hedw.) Schimp.

Loc: 112, 118, on soil, U2914; U2913;

Dicranum scoparium Hedw.

Loc: 106, 117, on soil, U2909; U2910;

Rhabdoweisiaceae Limpr.

**Dicranoweisia cirrata (Hedw.) Lindb.

Loc: 126; on oak bark, U3091;

Pottiaceae Schimp.

**Barbula convoluta Hedw.

Loc: 119, on bark, U3064; Loc: 133, on rock, $\mathrm{U} 3065$;

Barbula unguiculata Hedw.

Loc: 94, 133, on soil, U3064; U3067;

Didymodon ferrugineus (Schimp. ex Besch.)

M.O.Hill

Loc: 93, on concrete, U3052;

**Didymodon sinuosus (Mitt.) Delogne

Loc: 92, on soil, U3061; Loc: 117, on bark, $\mathrm{U} 3060$;

**Didymodon tophaceus (Brid.) Lisa

Loc: 105, 115, on soil, U3056;U3057;

**Gymnostomum aeruginosum $\mathrm{Sm}$.

Loc: 118, on $\log$, U3050;

**Leptobarbula berica (De Not.) Schimp.

Loc: 87, on soil, U3048;

*Stegonia latifolia (Schwägr.) Venturi ex Broth.

Loc: 94, on soil, U3049;

**Syntrichia laevipila Brid.

Loc: 85, 99, 101, on bark, U3076; U3074;

$\mathrm{U} 3075$;

*Syntrichia latifolia (Bruch ex Hartm.) Huebener

Loc: 101, on bark, U3045;

**Syntrichia papillosa (Wilson) Jur.

Loc: 99, on bark, U3046;

Syntrichia ruralis var. ruraliformis (Besch.)

Delogne

Loc: 78, on soil, U2803;

**Tortella flavovirens (Bruch) Broth.

Loc: 87, on bark, U3047;

**Tortella squarrosa (Brid.) Limpr.

Loc: 85, on soil, U3051;

*Tortula canescens Mont. 
Loc: 124, rock crevices, U3043;

**Tortula marginata (Bruch \& Schimp.) Spruce

Loc: 118, on soil, U3055;

**Tortula muralis Hedw.

Loc: 94, 122, on concrete, U3053; U3054;

**Tortula muralis var. aestiva Brid. ex Hedw.

Loc: 110, 117, on soil, U3077; U3078;

Tortula subulata Hedw.

Loc: 118, on soil, U3058; Loc: 128, on rock, U3059;

**Tortula truncata (Hedw.) Mitt.

Loc: 87, 95, on soil, U3084; U3083;

*Tortula vahliana (Schultz) Mont.

Loc: 89, on bark, U3044;

**Trichostomum brachydontium Bruch

Loc: 92, 103, 125, on soil, U3089; U3087; U3085; Loc: 94, 98, on rock, U3086; U3088;

**Weissia brachycarpa (Nees \& Hornsch.) Jur.

Loc: 94, 106, 108, 119, on soil, U3080; U3079; U3081; U3082;

**Weissia condensa (Voit) Lindb.

Loc: 105, 117, 128, on soil, U3072; U3073; U3071;

**Weissia controversa Hedw.

Loc: 91, 104, on soil, U3070; U3069; Loc: 123, on bark, U3068;

Weissia multicapsularis (Sm.) Mitt.,

Loc: 105, on soil, U3090;

**Weissia rutilans (Hedw.) Lindb.

Loc: 127, 129, on rock, U3063; U3062;

Bryaceae Schwägr.

**Bryum dichotomum Hedw.

Loc: 98, on soil, U2968; Loc: 128, on rock, U2967;

**Bryum gemmiparum De Not.

Loc: 100, on wet soil, U2962;

**Bryum subapiculatum Hampe

Loc: 87, 117, on soil, U2969; U2963,

Imbribryum alpinum (Huds. ex With.) N. Pedersen

Loc: 85, 86, 90, 106, on soil, U2977; U2978; U2979; U2976;

Ptychostomum capillare (Hedw.) Holyoak \& N. Pedersen

Loc: 88, 116, on decayed log, U2987; U2989; Loc: 108, 111, on soil, U2983; U2990; Loc: 87, 106, 117, U2988, U2986, U2984;

**Ptychostomum imbricatulum (Müll. Hal.) Holyoak \& N. Pedersen

Loc: 93, 88, 123, on soil, U2964; U2973; U2974;

Loc: 106, on bark, U2975;

Ptychostomum pallens (Sw.) J.R. Spence

Loc: 116, on bark, U2965;

**Ptychostomum pseudotriquetrum (Hedw.)

J.R.Spence \& H.P.Ramsay

Loc: 85, on soil, U2972;

Ptychostomum torquescens (Bruch \& Schimp.)

Ros \& Mazimpaka
Loc: 93, 117, on soil, U2982; U2981; Loc: 116, on bark, U2980;

Mniaceae Schwägr.

**Epipterygium tozeri (Grev.) Lindb.

Loc: 104, 131, on soil, U2970; U2971;

**Plagiomnium affine (Blandow ex Funck) T.J.

Kop.

Loc: 109, on rock, U2841;

**Plagiomnium elatum (Bruch \& Schimp.)

T.J.Kop.

Loc: 87, 109, on soil, U2844; U2842; Loc: 127, on rock, U2843;

Plagiomnium undulatum (Hedw.) T.J. Kop.

Loc: 114 , on soil, U2840;

Rhizomnium punctatum (Hedw.) T.J.Kop.

Loc: $114,119,131$, on soil, U2848;

U2847;U2846; Loc: 114, on rock, U2845;

Orthotrichaceae Arn.

Orthotrichum affine Schrad. ex Brid.

Loc: 91, 94, 101, 103, 106, 108, 109, 116, 119, 122, on bark, U2924; U2930; U2926; U2931; U2929; U2927; U2928; U2925; U2923; U2932;

**Orthotrichum anomalum Hedw.

Loc: 104, on rock, U2915;

**Orthotrichum diaphanum Brid.

Loc: 85, 92, 98, on bark, U2922; U2921; U2920;

**Orthotrichum lyellii Hook. \& Taylor (pulvigeria)

Loc: 110, on bark, U2917;

*Orthotrichum patens Bruch \& Brid.

Loc: 89 , on bark, U2916;

**Orthotrichum stramineum Hornsch. ex Brid.

Loc: 87, 99, on bark, U2919; U2918;

Amblystegiaceae G. Roth.

Amblystegium serpens var. juratzkanum (Schimp.) Rau \& Herv.

Loc: 101, on bark, U2831;

**Drepanocladus aduncus (Hedw.) Warn

Loc: 85 , on soil, U2830;

**Leptodictyum riparium (Hedw.) Warnst.

Loc: 90, on bark, U2833; Loc: on decayed log, U2834;

* Campylopus subulatus Schimp. ex Milde

Loc: 106, 121, on soil, U2901; U2912;

*Pseudoleskeella catenulata (Brid. ex Schrad.)

Kindb.

Loc: 125 , on soil, U2828;

**Pseudoleskeella rupestris (Berggr.) Hedenäs \& L. Söderstr.

Loc: 94, on rock, U2829;

Brachytheciaceae G. Roth.

- Brachythecium capillaceum (F.Weber \& D.Mohr) Giacom.

Loc: 93, on rock, U2995;

Brachythecium rutabulum (Hedw.)Schimp.

Loc: 87, 88, 100, on soil, U3019, U3020; U3018;

**Cirriphyllum crassinervium (Taylor) Loeske \& M.Fleisch. 
Loc: 104, on rock, U3014; Loc: 130, on log, U3013;

**Eurhynchium striatum (Hedw.) Schimp.

Loc: 95, on soil, U3000;

**Homalothecium lutescens (Hedw.) H. Rob. Loc: 90, 99, 119, on bark, U3029; U3026; U3027; Loc: 130, on rock, U3028;

**Homalothecium philippeanum (Spruce) Schimp.

Loc: 106, 126, on bark, U3002; U3003; Loc: 132, U3004;

Homalothecium sericeum (Hedw.) Schimp.

Loc: 93, 118, 128, on bark, U3016; U3015; U3017;

**Kindbergia praelonga (Hedw.) Ochyra

Loc: 90, 91, on bark, U3040; U3042; Loc: 118, on decayed log, U3038; Loc: 91, 94, on soil, U3041; U3036; Loc: 114, 122, on wet rock, U3039; U3001;

**Microeurhynchium pumilum (Wilson) Ignatov \& Vanderp.

Loc: 93, 94, 126, on soil, U3032; U3035; Loc: 93, on bark, U3034; Loc: 130, on rock, U3031; **Oxyrrhynchium schleicheri (R.Hedw.) Röll Loc: 86, 92, on soil, U3025; U3023; Loc: 103, on bark, U3024;

**Pseudoscleropodium purum (Hedw.) M. Fleisch.

Loc: 92, 125, on soil, U2994; U2999;

**Rhynchostegium confertum (Dicks.) Schimp.

Loc: 98, on bark, U2992; Loc: 103, 127, on rock, U2993, U2998;

*Sciuro-hypnum starkei (Brid.) Ignatov \& Huttunen

Loc: 92, 123, on soil, U3012, U3011;

**Scleropodium cespitans (Müll. Hal.) L.F. Koch

Loc: 94, 123, on soil, U3009; U3007; Loc: 93, 127, on rock, U3008, U3010;

**Scleropodium touretii (Brid.) L.F. Koch

Loc: 93, on rock, U3022;

Hypnaceae Schimp.

**Calliergonella cuspidata (Hedw.) Loeske

Loc: 91, on soil, U2832;

**Hypnum cupressiforme Hedw. var. cupressiforme

Loc: 106, 111, 117, on bark, U2960; U2961; Loc: 94, on soil, U2956; Loc: 121, 131, on decayed log, U2958; U2959;

**Hypnum cupressiforme Hedw. var. filiforme Brid.

Loc: 94, 109, 116, 119, 128, on bark, U2941; U2945; U2943; U1942; U2944;

**Hypnum cupressiforme Hedw. var. lacunosum Brid.

Loc: 87, 123, on bark, U2952; U2950; Loc: 90, on log, U2949; Loc: 117, on soil, U2953;
**Hypnum cupressiforme Hedw. var. resupinatum (Taylor) Schimp.

Loc: 87, 106, 110, on bark, U2947; U2948; U2946;

*Hypnum cupressiforme Hedw. var. subjulaceum Molendo

Loc: 85, on bark, U2955; Loc: 117, on soil, $\mathrm{U} 2954$;

**Hypnum imponens Hedw.

Loc: 108, on bark, U2940;

- Pseudotaxiphyllum elegans (Brid.) Z.Iwats

Loc: 111, on bark, U2935;

Hylocomiaceae M. Fleisch.

**Ctenidium molluscum (Hedw.) Mitt

Loc: 120, on roil, U2475;

Plagiotheciaceae (Broth.) M. Fleisch.

**Plagiothecium cavifolium (Brid.) Z.Iwats.

Loc: 111, 112, on bark, U2938, U2937;

**Plagiothecium laetum Schimp.

Loc: 113, 131, on soil, U2934; U2936;

Cryphaeaceae Schimp.

**Cryphaea heteromalla (Hedw.) D. Mohr

Loc: 92, 99, on bark, U2838; U2839;

Leucodontaceae Schimp.

**Antitrichia curtipendula (Hedw.) Brid.

Loc: 109, 117, on bark, U2991; U2866;

Leucodon sciuroides (Hedw.) Schwägr.

Loc: 87, 90, 99, 100, 113, on bark, U2868; U2837; U2870; U2867;

Leptodontaceae Schimp.

**Leptodon smithii (Hedw.) F.Weber \& D.Mohr Loc: 89, 93, 99, 126, on bark, U2877; U2875; U2874; U2878; Loc: 103, on rock, U2876;

Neckeraceae Schimp.

**Alleniella besseri (Lobarz.) S.Olsson, Enroth \& D.Quandt

Loc: 103, 131, on rock, U2872; U2873;

**Alleniella complanata (Hedw.) S. Olsson, Enroth \& D.Quandt

Loc: 112, on bark, U2879; Loc: 132, on soil, $\mathrm{U} 2880$;

**Neckera pumila Hedw.

Loc: 99, on bark, U2871;

Thamnobryum alopecurum (Hedw.) Gangulee

Loc: 103, 127, on rock, U2883, U2885; Loc: 130, on soil, U2884;

Lembophyllaceae Broth.

Isothecium alopecuroides (Lam. ex Dubois) Isov.

Loc: 93, on bark, U3006; Loc: 113, on rock, U3005;

**Isothecium holtii Kindb.

Loc: 108, on rock, U2996; Loc: 111, on bark, U2997;

Anomodontaceae Kindb.

**Anomodon viticulosus (Hedw.) Hook. \& Taylor

Loc: 131, on rock, U2882; 


\section{Results and Discussion}

As a results of bryofloristic study in the area; in a total 112 taxa belonging to 22 families and 60 genera were determined. Among them Weissia multicapsularis (Sm.) Mitt. (Figure 2) was recorded the first time from Turkey with the this study (Ursavaş and Keçeli, 2018). And also Pseudotaxiphyllum elegans (Brid.) Z.Iwats and Brachythecium capillaceum (F.Weber \& D.Mohr) Giacom are recorded for the second time from Turkey.

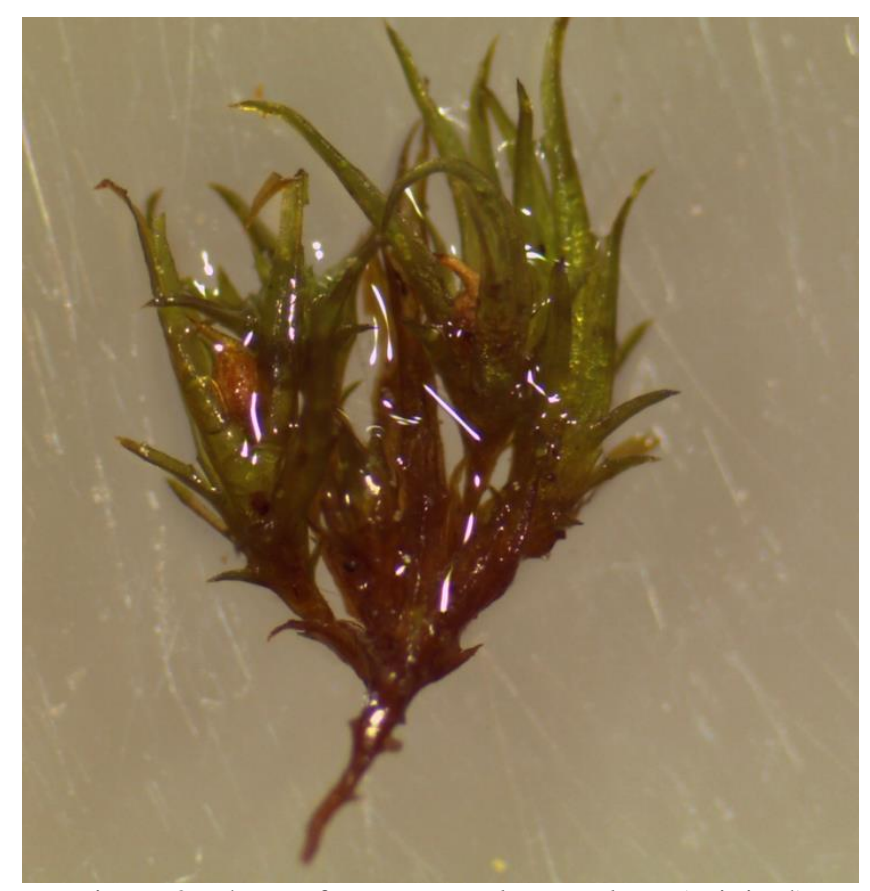

Figure 2. Photo of Weissia multicapsularis (original)

Furthermore, according to the Henderson (1961)'s Turkey grid system, 13 of these taxa are new records for the A1 grid square: Atrichum tenellum (Röhl.) Bruch \& Schimp., Ceratodon conicus (Hampe) Lindb., Campylopus subulatus Schimp. ex Milde, Dicranella subulata (Hedw.) Schimp., Fissidens viridulus var. viridulus ( $\mathrm{Sw}$. ex anon.) Wahlenb., Orthotrichum patens Bruch \& Brid., Pseudoleskeella catenulata (Brid. ex Schrad.) Kindb., Stegonia latifolia (Schwägr.) Venturi ex Broth., Sciuro-hypnum starkei (Brid.) Ignatov \& Huttunen, Syntrichia latifolia (Bruch ex Hartm.) Huebener, Tortula canescens Mont., Tortula vahliana (Schultz) Mont., Hypnum cupressiforme Hedw. var. subjulaceum Molendo (Çetin and Uyar, 1997; Çetin, 1999; Uyar and Çetin, 2006; Keçeli and Çetin, 2006; Ören et al., 2010, 2012; Keçeli et al., 2012; Ros et al., 2013).
So far, there has been only two bryophyte study in the province of Bursa (Çetin, 1999 and Can Gözcü et al., 2018). Bryophyte studies in Bursa were examined and 69 taxa from these studies are new records for the Bursa province.

According to Table 1. the richest families in terms of a number of taxa, respectively, are: Pottiaceae (27), Brachytheciaceae (15), Bryaceae (9), Hypnaceae (8), Amblystegiaceae (6), Orthotrichaceae (6), Polytrichaceae (5), Mniaceae (5). Tortula (Pottiaceae) is the most species rich genus in the sutdy area. The present study compared with the other studies that are closest to the study area. The comparison of the floristic studies according to the families is given in Table 2 and the genus level is given in Table 3. 
Table 1 . The distributions of taxa on the family level in the study area

\begin{tabular}{|c|c|c|c|c|}
\hline $\begin{array}{c}\text { Family } \\
\text { No }\end{array}$ & Family & The number of genus & Genus & The number of taxa \\
\hline \multirow{11}{*}{1} & \multirow{11}{*}{ Brachytheciaceae } & \multirow{11}{*}{11} & Brachythecium & 2 \\
\hline & & & Cirriphyllum & 1 \\
\hline & & & Eurhynchium & 1 \\
\hline & & & Homalothecium & 3 \\
\hline & & & Kindbergia & 1 \\
\hline & & & Microeurhynchium & 1 \\
\hline & & & Oxyrrhynchium & 1 \\
\hline & & & Pseudoscleropodium & 1 \\
\hline & & & Rhynchostegium & 1 \\
\hline & & & Sciuro-hypnum & 1 \\
\hline & & & Scleropodium & 2 \\
\hline \multirow{10}{*}{2} & \multirow{10}{*}{ Pottiaceae } & \multirow{10}{*}{10} & Barbula & 2 \\
\hline & & & Didymodon & 3 \\
\hline & & & Gymnostomum & 1 \\
\hline & & & Leptobarbula & 1 \\
\hline & & & Stegonia & 1 \\
\hline & & & Syntrichia & 4 \\
\hline & & & Tortella & 2 \\
\hline & & & Tortula & 7 \\
\hline & & & Trichostomum & 1 \\
\hline & & & Weissia & 5 \\
\hline \multirow{5}{*}{3} & \multirow{5}{*}{ Amblystegiaceae } & \multirow{5}{*}{5} & Amblystegium & 1 \\
\hline & & & Drepanocladus & 1 \\
\hline & & & Leptodictyum & 1 \\
\hline & & & Campylopus & 1 \\
\hline & & & Pseudoleskeella & 2 \\
\hline \multirow{3}{*}{4} & \multirow{3}{*}{ Polytrichaceae } & \multirow{3}{*}{3} & Atrichum & 2 \\
\hline & & & Pogonatum & 1 \\
\hline & & & Polytrichum & 2 \\
\hline \multirow{3}{*}{5} & \multirow{3}{*}{ Ditrichaceae } & \multirow{3}{*}{3} & Ceratodon & 2 \\
\hline & & & Ditrichum & 1 \\
\hline & & & Pleuridium & 1 \\
\hline \multirow{3}{*}{6} & & & Bryum & 3 \\
\hline & Bryaceae & 3 & Imbribryum & 1 \\
\hline & & & Ptychostomum & 5 \\
\hline & & & Epipterygium & 1 \\
\hline 7 & Mniaceae & 3 & Plagiomnium & 3 \\
\hline & & & Rhizomnium & 1 \\
\hline & & & Calliergonella & 1 \\
\hline 8 & Hypnaceae & 3 & Hyрпит & 6 \\
\hline & & & Pseudotaxiphyllum & 1 \\
\hline & & & Alleniella & 2 \\
\hline 9 & Neckeraceae & 3 & Neckera & 1 \\
\hline & & & Thamnobryum & 1 \\
\hline 10 & Grimmiaceae & 2 & Grimmia & 3 \\
\hline 10 & Grimmiaceae & 2 & Schistidium & 1 \\
\hline 11 & Dicranaceae & 2 & Dicranella & 2 \\
\hline 11 & Dicranaceae & 2 & Dicranum & 1 \\
\hline 12 & Leucodontaceae & 2 & Antitrichia & 1 \\
\hline 12 & Leucodontaceae & 2 & Leucodon & 1 \\
\hline 13 & Orthotrichaceae & 1 & Orthotrichum & 6 \\
\hline 14 & Hylocomiaceae & 1 & Ctenidium & 1 \\
\hline 15 & Plagiotheciaceae & 1 & Plagiothecium & 2 \\
\hline 16 & Cryphaeaceae & 1 & Cryphaea & 1 \\
\hline 17 & Leptodontaceae & 1 & Leptodon & 1 \\
\hline 18 & Funariaceae & 1 & Funaria & 1 \\
\hline 19 & Fissidentaceae & 1 & Fissidens & 4 \\
\hline 20 & Rhabdoweisiaceae & 1 & Dicranoweisia & 1 \\
\hline 21 & Lembophyllaceae & 1 & Isothecium & 2 \\
\hline 22 & Anomodontacea & 1 & Anomodon & 1 \\
\hline & TOTAL & 60 & & 112 \\
\hline
\end{tabular}


Table 2. Comparison with the families in some bryophyte flora studies in A1 square.

\begin{tabular}{|c|c|c|c|c|c|c|c|c|}
\hline \multirow{2}{*}{$\begin{array}{l}\text { Bryophyte Studies } \\
\text { Families }\end{array}$} & \multicolumn{2}{|c|}{$\begin{array}{c}\text { The Moss Flora } \\
\text { of Kocaçay Delta } \\
\text { Floodplain } \\
\text { Forest in Turkey } \\
(2019)\end{array}$} & \multicolumn{2}{|c|}{ 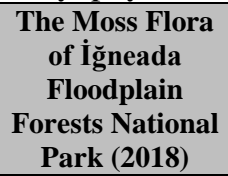 } & \multicolumn{2}{|c|}{$\begin{array}{c}\text { Contribution to } \\
\text { The Bryophyte } \\
\text { Flora of } \\
\text { European (2008) }\end{array}$} & \multicolumn{2}{|c|}{$\begin{array}{l}\text { Contribution to } \\
\text { The Bryophyte } \\
\text { Flora of Turkish } \\
\text { Thrace (2003) }\end{array}$} \\
\hline & $\begin{array}{c}\text { The } \\
\text { number } \\
\text { of taxa }\end{array}$ & $\%$ & $\begin{array}{c}\text { The } \\
\text { number } \\
\text { of taxa }\end{array}$ & $\%$ & $\begin{array}{c}\text { The } \\
\text { number } \\
\text { of taxa }\end{array}$ & $\%$ & $\begin{array}{c}\text { The } \\
\text { number } \\
\text { of taxa }\end{array}$ & $\%$ \\
\hline Pottiaceae & 27 & 24 & 20 & 19 & 33 & 26.2 & 46 & 36.8 \\
\hline Brachytheciaceae & 15 & 13 & 14 & 13.3 & 19 & 15.1 & 18 & 14.4 \\
\hline Bryaceae & 9 & 8 & 6 & 5.7 & 14 & 11.1 & 11 & 8.8 \\
\hline Hypnaceae & 8 & 7 & 6 & 5.7 & 3 & 2.4 & 4 & 3.2 \\
\hline Amblystegiaceae & 6 & 5.3 & 2 & 1.9 & 5 & 4 & 4 & 3.2 \\
\hline Orthotrichaceae & 6 & 5.3 & 9 & 8.6 & 10 & 7.9 & 10 & 8 \\
\hline Polytrichaceae & 5 & 4.4 & 9 & 8.6 & 4 & 3.2 & 4 & 3.2 \\
\hline Mniaceae & 5 & 4.4 & 4 & 3.9 & 4 & 3.2 & 1 & 0.8 \\
\hline Fissidentaceae & 4 & 4.5 & 4 & 3.9 & 7 & 5.6 & - & - \\
\hline Neckeraceae & 4 & 4.5 & 2 & 1.9 & 3 & 2.4 & - & - \\
\hline Grimmiaceae & 4 & 4.5 & 4 & 3.9 & 5 & 4 & 4 & 3.2 \\
\hline Dicranaceae & 4 & 4.5 & 2 & 1.9 & 2 & 1.6 & 4 & 3.2 \\
\hline Ditrichaceae & 3 & 2.6 & 2 & 1.9 & 5 & 4 & 5 & 4 \\
\hline Leucodontaceae & 2 & 1.7 & 2 & 1.9 & 2 & 1.6 & 1 & 0.8 \\
\hline Plagiotheciaceae & 2 & 1.7 & 2 & 1.9 & 2 & 1.6 & - & - \\
\hline Lembophyllaceae & 2 & 1.7 & 1 & 0.9 & 1 & 0.8 & 1 & 0.8 \\
\hline Hylocomiaceae & 1 & 0.8 & - & - & - & - & - & - \\
\hline Cryphaeaceae & 1 & 0.8 & 1 & 0.9 & - & - & - & - \\
\hline Leptodontaceae & 1 & 0.8 & - & - & - & - & - & - \\
\hline Funariaceae & 1 & 0.8 & 4 & 3.9 & 1 & 0.8 & 1 & 0.8 \\
\hline Anomodontacea & 1 & 0.8 & 2 & 1.9 & 1 & 0.8 & - & - \\
\hline Rhabdoweisiaceae & 1 & 0.8 & - & - & - & - & - & - \\
\hline
\end{tabular}

Table 3. Compared with the genus in some bryophyte flora studies in A1 square

\begin{tabular}{|l|c|c|c|c|c|c|c|c|}
\hline & $\begin{array}{c}\text { The Moss Flora } \\
\text { of Kocaçay } \\
\text { Delta } \\
\text { Bloodplain } \\
\text { Forest in } \\
\text { Turkey (2019) }\end{array}$ & $\begin{array}{c}\text { The Moss Flora } \\
\text { of İgneada } \\
\text { Floodplain } \\
\text { Forests National } \\
\text { Park (2018) }\end{array}$ & $\begin{array}{c}\text { Contribution to } \\
\text { The Bryophyte } \\
\text { Flora of } \\
\text { European (2008) }\end{array}$ & $\begin{array}{c}\text { Contribution to } \\
\text { The Bryophyte } \\
\text { Flora of Turkish } \\
\text { Thrace (2003) }\end{array}$ \\
\hline Families & $\begin{array}{c}\text { The } \\
\text { number } \\
\text { of taxa }\end{array}$ & \% & $\begin{array}{c}\text { The } \\
\text { number } \\
\text { of taxa }\end{array}$ & \% & $\begin{array}{c}\text { The } \\
\text { number } \\
\text { of taxa }\end{array}$ & \% & $\begin{array}{c}\text { The } \\
\text { number } \\
\text { of taxa }\end{array}$ & \% \\
\hline Tortula & 7 & 6.2 & 5 & 4.8 & 5 & 4 & 15 & 12 \\
\hline Hypnum & 6 & 5.4 & 4 & 3.8 & 2 & 1.6 & 2 & 1.6 \\
\hline Orthotrichum & 6 & 5.4 & 9 & 8.6 & 9 & 7.1 & 9 & 7.2 \\
\hline Weissia & 5 & 4.4 & 4 & 3.8 & 3 & 2.4 & 4 & 3.2 \\
\hline Ptychostomum & 5 & 4.4 & 3 & 2.9 & - & - & - & - \\
\hline Syntrichia & 4 & 3.6 & 4 & 3.8 & 5 & 4 & - & - \\
\hline Fissidens & 4 & 3.6 & 4 & 3.8 & 7 & 5.6 & 10 & 8 \\
\hline Homalothecium & 3 & 2.6 & 2 & 1.9 & 4 & 3.2 & 3 & 2.4 \\
\hline Didymodon & 3 & 2.6 & - & - & - & - & - & - \\
\hline Bryum & 3 & 2.6 & 2 & 1.9 & 14 & 11 & 11 & 8.8 \\
\hline Dicranella & 3 & 2.6 & 1 & 1 & 1 & 0.8 & 2 & 1.6 \\
\hline Grimmia & 3 & 2.6 & 3 & 2.9 & 2 & 1.6 & 3 & 2.4 \\
\hline Plagiomnium & 3 & 2.6 & 3 & 2.9 & 1 & 0.8 & - & - \\
\hline Brachythecium & 2 & 1.8 & 4 & 3.8 & 3 & 2.4 & 3 & 2.4 \\
\hline Scleropodium & 2 & 1.8 & 2 & 1.9 & 1 & 0.8 & 3 & 2.4 \\
\hline Barbula & 2 & 1.8 & 2 & 1.9 & 1 & 0.8 & 2 & 1.6 \\
\hline Tortella & 2 & 1.8 & - & - & - & - & - & - \\
\hline Pseudoleskeella & 2 & 1.8 & - & - & - & - & - & - \\
\hline Atrichum & 2 & 1.8 & 4 & 3.8 & 2 & 1.6 & - & - \\
\hline Polytrichum & 2 & 1.8 & 3 & 2.9 & 2 & 1.6 & 1 & 0.8 \\
\hline Ceratodon & 1.8 & - & - & - & - & - & - \\
\hline Alleniella & 2 & - & - & - & - & - & - \\
\hline
\end{tabular}


According to Table 2, it is expected that the families of Pottiaceae and Brachytheciaceae will share the first two places. The Orthotrichaceae family is highly represented in the European continent (9-10), while the Kocaçay Delta (6) remains low (Asia). This may be due to continental differences or tree species diversity and age differences.

According to Table 3, there are differences in the number of taxa determined from research areas. This can probably be related to the size of the study areas.

Acknowledgements: This study was financially supported by the Scientific and Technological Research Council of Turkey (TÜBITAK) (Project No: 115Z364). We would like to thank to Richard H. Zander and Des Callagha for his help to confirm the determination of Weissia multicapsularis.

Annotation: This study was presented as an Oral Presentation at the International Symposium of Ecology 19-23 June 2018 in Kastamonu, Turkey, and published in the Abstract Book of the Symposium.

\section{References}

Aleffi M. Tacchi R. Cortini Pedrotti C. 2008. Check-list of Hornworts, Liverworts and Mosses of Italy. Bocconea. 22: 5-254.

Corbière L. 1905. Musciǹèes des environs de Constantine. Comptes rendus de lássociation Française pour lávancement des sciences. pp. 487.

Crum H. 1973. Mosses of the Great Lakes forest.Üniversity of Michigan. Michigan, Amerika. pp. 404.

Crum H.A. Anderson L.E. 1981. Mosses of Eastern North America. Vol. 1-2., New York: Columbia University Press.

Çetin B. 1999. The Moss Flora of the Uludağ National Park (Bursa/Turkey), Ankara Üniversitesi Biyoloji Anabilim Dalı. Ankara. pp. 193.

Çetin B. Uyar G. 1997. The Moss Flora of Sinop and Its Environs (Ayancik, Boyabat and Gerze). Turkish Journal of Botany. 21: 231-244.

Eken G. Bozdoğan M. İsfendiyaroğlu S. Kılıç D.T. Lise Y. 2006. Türkiye'nin Önemli Doğa Alanları. Doğa Derneği. Ankara.

Greven H.C. 2003. Grimmias of The World. Leiden: Backhuys Publishers. The Netherlands. pp. 250.

Henderson D.M. 1961. Contributions to the Bryophyte Flora of Turkey: IV. Notes from the Royal Botanic Garden Edinburgh, 23: 263-278.

Heyn C.C. Herrnstadt I. 2004. The Bryophyte Flora of Israel and Adjacent Regions. The Israel Academy of Sciences and Humanities, Jerusalem. pp. 719.

Hodgetts N.G. 2015. Checklist and contry status of European bryophytes towards a new Red List for Europe. Irish Wildlife Manuals, No. 84. National Parks and Wildlife Service, Department of Arts, Heritage and the Gaeltacht, Ireland.

Işın Z. Ursavaş S. 2018. The Moss Flora of İğneada Floodplain Forests National Park (Demirköy, Kırklareli) Turkey. Anatolian Bryology. 4:2, 92-106.

Keçeli T. Çetin B. 2006. A Contribution to the Liverwort Flora of Western Black Sea Region, Northern Turkey, and a new record (Cephaloziella dentata, Cephaloziellaceae) to Southwest Asia. Cryptogamie Bryologie. 27:4, 459-470.

Kavgaci A. Özalp G. Özhayat N. 2007. Flora of Igneada Fioodplain Forests (Longozes) and Their Surroundings. JFFIU. 57: 6089.

Keçeli T. Ören M. Uyar G. 2012. Türkiye Ciğerotları (Marchantiophyta) Florası için İki Yeni Kayıt: Cephalozia lunulifolia (Cephaloziaceae) ve Lophocolea fragrans (Lophocoleaceae), 21. Ulusal Biyoloji Kongresi, Bildiriler Kitab1. PB 161, ss. 576-577. 3-7 Eylül 2012, İzmir, Özetler Kitab1. pp. 576-577.

Lara F. Garilleti R. Goffinet B. Draper I. Medina R. Vigalondo B. Mazimpaka V. 2016. Lewinskya, a New Genus to Accommodate the Phaneroporous and Monoicous Taxa of Orthotrichum (Bryophyta, Orthotrichaceae). Cryptogamie, Bryologie. 37:4, 361-382.

Lawton E. 1971. Moss Flora of Pasific Northwest. Journal of Hattori Botanical Garden Laboratory, Nichinan. pp. 760. Lockhart N.D. Hodgetts N.G. Holyoak D.T. 2012. Rare and threatened bryophytes of Ireland. National Museums Northern Ireland, Holywood, Co Down.

Lüth M. 2006. Bildatlas der Moose Deutschlands, Faszikel 1. Grimmiaceae. Freiburg. Deutschland.

Lüth M. 2006. Bildatlas der Moose Deutschlands, Faszikel 2. Dicranaceae Miniaceae - Politrichaceae. Freiburg. Deutschland.

Lüth M. 2006. Bildatlas der Moose Deutschlands, Faszikel 3. Pottiaceae. Freiburg. Deutschland. 
Lüth M. 2007. Bildatlas der Moose Deutschlands, Faszikel 4. Bryaceae Disceliaceae - Ephemeraceae Funariaceae - Splachnaceae. Freiburg. Deutschland.

Lüth M. 2008. Bildatlas der Moose Deutschlands, Faszikel 5. Timmiaceae. Freiburg. Deutschland.

Lüth M. 2009. Bildatlas der Moose Deutschlands, Faszikel 6. Amblystegiaceae - Thuidiaceae. Freiburg. Deutschland.

Lüth M. 2010. Bildatlas der Moose Deutschlands, Faszikel 7. Brachytheciaceae - Entodontaceae Hypnaceae - Plagiotheciaceae Sematophyllaceae. Freiburg. Deutschland.

Ören M. Uyar G. Keçeli T. 2010. Anomodon longifolius (Anomodontaceae, Bryopsida) New To The Bryophyte Flora of Turkey. Turkish Journal of Botany. 34: 141-145.

Ören M. Uyar G. Keçeli T. 2012. The bryophyte flora of the western part of the Küre Mountains (Bartın, Kastamonu), Turkey. Turkish Journal of Botany. 36: 538-557.

Pedrotti C.C. 2001. Flora dei muschi d'Italia, Sphagnopsida, Andreaopsida, Bryopsida (I parte). Antonio Delfino Editore Medicina-Scienze. pp. 817.

Pedrotti C.C. 2006. Flora dei muschi d'Italia, Bryopsida (II parte). Roma: Antonia Delfino Editore. Antonio Delfino Editore Medicina-Scienze. pp. 817-1235.

Plášek V. Sawicki J. Ochyra R. Szczeciñska M. Kulik T. 2015. New taxonomical arrangement of the traditionally conceived genera Orthotrichum and Ulota (Orthotrichaceae, Bryophyta). Acta Musei Silesiae, Scientiae Naturales. 64: 169-174.

Porley R. 2013. England's rare mosses and liverworts. Princeton (NJ), Woodstock (UK): Princeton University Press.

Ros R.M. Mazimpaka V. Abou-Salama U. Aleffi M. Blockeel T.L. Brugués M. Cros R.M. Dia M.G. Dirkse G.M. Draper I. et al., 2013. Mosses of the Mediterranean, an annotated checklist. Cryptogamie Bryolgie. 34: 99-283.

Saçın Y. 2010. Kocaçay Deltası ve Uluabat Gölünün Uzaktan Algılama Metodları Kullanılarak İncelenmesi. s. 92. Yüksek Lisans Tezi.

Schuck A. Parviainen J. Bücking W. 1994. A review of approaches to forestry research on structure, succession and biodiversity of a disturbed and semi-natural forests and woodland in Europe. European Forest Institute Working Paper 3, Joensuu, pp. 64.

Smith A.J.E. 1980. The Moss Flora of Britain and Ireland.Cambridge University Press. pp. 706.

Smith A.J.E. 2004. The Moss Flora of Britain an Ireland.Cambridge University Press. pp. 1012.

URL1.Bursa-Karacabey Ovakorusu Celal Acar Yaban Hayvanları Kurtarma ve Rehabilitasyon Merkezi. 2019. Website: http://bolge2.ormansu.gov.tr/2bolge/AnaS ayfa/CelalAcarYabanHayati.aspx?sflang= tr [Accessed: 23 January 2019].

URL2.Karacabey Longoz Ormanlar1. 2019. Website:

http://www.bursa.com.tr/karacabeylongozu-576.html [Accessed: 22 January 2019].

Ursavaş S. Keçeli T. 2018. Weissia multicapsularis, a rare moss species new to Turkey and Asia. Plant Biosystems. 14. Doi: 10.1080/11263504.2018.1536086.

Uyar G. Çetin B. 2006. Contribution to the moss flora of Turkey: Western Black Sea Region (Bolu, Katamonu, Karabük, Bartın and Zonguldak). International Journal of Botany. 2:3, 229-241.

Watson E. V. 1981. British Mosses and Liverworts. Cambridge: Cambridge University Press.

Wenger E. Zinke A. Gutzweiler K.A. 1990. Present Situation of the European Floodplain Forests. For Ecology and Management. 33:34, 5-12. 